\title{
Geschichte und Erinnerung der NS-Zwangsarbeit als lebensgeschichtlich reflektierte Arbeitserfahrung
}

\section{Einleitung: "Cultural discourse about what has happened" (Lutz Niethammer)}

Im Jahre 2000 beschloss der deutsche Bundestag historisch erstmalig individuell bemessene und von den Betroffenen selbst zu beantragende Wiedergutmachungszahlungen an ehemalige NS-Zwangsarbeitende, die während des Zweiten Weltkriegs für Deutschland erzwungene Arbeitsleistungen erbracht hatten. Es gab zwei Hauptgruppen leistungsberechtigter Personen, die anhand der besonderen Bedingungen ihres zwangsweisen Arbeitseinsatzes unterteilt wurden, sowie eine dritte, die gemäß der Art der Schädigung (Vermögenseinbußen) konzipiert wurde. ${ }^{1}$

Die erste der beiden Hauptgruppen, die für diesen Beitrag relevant sind, umfasste ehemals in Konzentrationslagern oder ,vergleichbaren Haftstätten“ internierte Personen, die aufgrund der besonderen Schwere ihrer Internierungsumstände in der historischen Forschung und im Entschädigungsdiskurs als Sklavenarbeiter bezeichnet werden. Entsprechend dieser Umstände erhielten sie deutlich mehr Geld als die zweite Hauptgruppe der zivilen Deportierten, für die im engeren Sinne der Begriff Zwangsarbeiter benutzt wird. Kriegsgefangene wurden zwar im Gesetz als weitere Gruppe erwähnt, waren aber trotz Erbringung erzwungener Arbeitsleistungen grundsätzlich von den Zahlungen ausgenommen.

Mit Blick auf die in diesem Verfahren anhand von Umständen des Gewahrsams, der Verbringung und des Rechtsstatus erstellten Kategorien zur Einteilung und Eingrenzung des Kreises der Bezugsberechtigten von Entschädigungsleistungen hatte Niethammer bereits 2001 gefordert: Diese „Kategorien müssen wieder in historische Verhältnisse aufgelöst werden,“ um die so (wieder)-

1 Hierzu und zum Folgenden: Gesetz zur Errichtung einer Stiftung „Erinnerung, Verantwortung und Zukunft“ vom 2.8.2000, in: BGBl. I, S. 1263, zuletzt durch Artikel 1 des Gesetzes vom 1.9.2008 (BGBl. I, S. 1797) geändert worden. 
gefundenen „Begriffe für die Zukunft erinnerungsfähig zu machen.“2 Dazu werden in diesem Beitrag Formen von Zwangsarbeit in der NS-Zeit in einen Deutungszusammenhang gestellt, der durch individualisierte und kollektivierte Erinnerungen seine besondere Perspektive erhält. Zunächst soll anhand der Beschreibung der Erinnerungen an Arbeitserfahrungen der verschiedenen Statusgruppen die Sicht der Betroffenen genauer herausgearbeitet werden. Daran anschließend werden die verschiedenen Formen des Zwangs, die die Alltagsrealität der NS-Zwangsarbeitenden charakterisierten, begrifflich neu konzipiert. Dazu lassen sich im Kontext der Arbeit von Ausländern und aus der „Volksgemeinschaft“ ausgeschlossener Deutscher mit Deportation bzw. Dislozierung, Internierung bzw. Gefangenschaft und unmittelbar bzw. schrittweise drohender Vernichtung verschiedene Abstufungen von Zwang und Gewalt ausmachen, mit denen verschiedene Arten von Bedrohungen einhergingen. Diese reichen von der Infragestellung individueller und sozialer Identitäten oder des beruflichen bzw. sozialen Status und des damit zusammenhängenden Prestiges über unmittelbare Eingriffe in die räumliche und körperliche Integrität der Betroffenen bis hin zur Inkaufnahme oder sogar vorsätzlichen Herbeiführung des Todes. Mit dieser Erschließung des Erfahrungs- und Deutungsraums hinsichtlich der verschiedenen Grade des Zwangs und der Formen von Bedrohung bzw. Beeinträchtigung werden die unter dem Begriff NS-Zwangsarbeit zusammengefassten Phänomene in ihrer jeweils spezifischen Qualität deutlicher erkennbar.

\section{Biographische Quellen als Mittel der Perspek- tivdifferenzierung in der historischen Forschung}

Zur NS-Zwangsarbeit liegen mittlerweile etliche Forschungen vor, auch biographische Perspektiven der Betroffenen sind dabei in jüngster Zeit verstärkt zur Geltung gekommen. ${ }^{3}$ Allerdings unterliegt die Darstellung des konkreten Vollzugs der Arbeit, vor allem im Zusammenhang der Debatten um individuelle Kompensationsleistungen seit Mitte der 1990er Jahre, starken normativen und politischen Vorannahmen, die den Blick auf das alltägliche Geschehen erzwungener Arbeitsleistungen für NS-Deutschland erschweren. Dieser Blick soll an-

2 Lutz Niethammer, Klärung und Aufklärung. Aufgaben und Lücken der Zwangsarbeiterforschung, in: Stiftung Bibliothek des Ruhrgebiets (Hrsg.), Zwangsarbeiterforschung als gesellschaftlicher Auftrag, Bochum 2001, S. 13-22, hier S. 20.

3 Z. B.: Alexander von Plato et al. (Hrsg.), Hitler's Slaves. Life Stories of Forced Labourers in Nazi-Occupied Europe, Oxford/New York 2010. 
hand einer kritischen Lektüre von Transkriptionen lebensgeschichtlicher Interviews mit ehemaligen NS-Zwangsarbeitenden, die im Rahmen des vom Institut für Geschichte und Biographie (IGB) der Fernuniversität Hagen in Verbindung mit 33 Institutionen in 26 Ländern von 2004 bis 2007 durchgeführten „International Forced Labourers' Documentation Project“ (IFLDP) entstanden sind ${ }^{4}$, geschärft werden. Für diesen Beitrag wurden dazu vor allem Interviews mit Personen, die heute in Deutschland, Frankreich, Großbritannien, Israel, Tschechien und der Ukraine leben, herangezogen. Die Interviews entstanden in zeitlicher Nähe zu den administrativen Entschädigungsverfahren und unter institutioneller Förderung der Stiftung „Erinnerung, Verantwortung und Zukunft“, was sie in ein konkretes erinnerungspolitisches und diskursives Bedingungsgefüge einbettet. Die in den Transkriptionen schriftlich niedergelegten, innerhalb lebensgeschichtlicher Interviews mündlich ausgedrückten Erinnerungen sind keine getreuen Abbilder vormaliger historischer Erfahrungen und unterlagen in unterschiedlichem Ausmaß nachträglicher narrativer bzw. diskursiver Überformung, bevor sie im Gesamtzusammenhang der je individuellen Lebensgeschichte als NS-Zwangsarbeitserfahrung formuliert wurden. Allerdings setzt sich dieser Beitrag auch bewusst ab von einer mittlerweile im wissenschaftlichen Diskurs Hegemonie beanspruchenden Haltung ab, die das Quellenprestige mündlicher autobiographischer Zeugnisse dahingehend fundamental $\mathrm{zu}$ diskreditieren versucht, als diese einerseits vollständig von zeitgenössischen Erinnerungsdiskursen, persönlichen Identitätsbedürfnissen sowie den Wahrnehmungs- und Werthorizonten der Gegenwart der Interviewten geprägt seien und andererseits keinerlei Bezug zu vergangenen Ereignissen herstellen könnten.

Die vorgebrachten Einwände sind für sich genommen ernst zu nehmen, bedürfen allerdings für eine Gesamteinschätzung auch der Relativierung. Die Literaturwissenschaftlerin Susan Suleiman hat in ihrer Untersuchung $\mathrm{zu}$ „Crises of Memory“ im Anschluss an Arbeiten des Literaturwissenschaftlers Michael Bernstein konstatiert, „... no first person narrative is 'untouched by figuration and by shaping' “" Dies geschieht auch nicht erst im Moment des Erzählens. Erfahrungen werden vielmehr schon im Vorgang des bewussten Erlebens diskursiv semantisiert. Es bleibt aber festzuhalten, dass selbst bei Annahme eines relationalen, in vielfältige Diskurse eingebundenen „dezentierten Subjekts“ (Philipp Sarasin) dieses doch weiterhin als maßgeblicher Akteur von Erinnerungsvor-

4 Dem Leiter des Projekts, Alexander von Plato, gilt Dank für wichtige Hinweise und Anmerkungen zu diesem Beitrag. Der Gesamtbestand der Interviews ist mittlerweile in einem passwortpflichtigen Internetportal der FU Berlin weiter erschlossen und digital aufbereitet worden: [http://www.zwangsarbeit-archiv.de/], eingesehen 26.1.2014.

5 Susan Suleiman, Crises of memory and the Second World War, Cambridge/MA 2006, S. 168. 
gängen anzusehen ist. Diese individualisierten Erinnerungen stehen zwar mit kollektiven Gedächtnisformen in Beziehung, bleiben ihnen gegenüber aber letztlich inkommensurabel oder wie es der Historiker Reinhart Koselleck mit einer Perspektive auf individuelle Erinnerung als eigensinniges Refugium ausgedrückt hat: „Der Mensch hat das Recht auf seine eigene Erinnerung - die lasse ich mir nicht kollektivieren." 6 So entwickeln sich zwischen individualisierten Erinnerungen von an historischen Begebenheiten Beteiligten und den korrespondierenden hegemonialen (Erinnerungs)Diskursen jeweiliger Gesellschaften erhebliche Spannungen und Inkongruenzen, die auch zu einer gegenseitigen Infragestellung, im besten Fall zu einer gegenseitigen produktiven Relativierung führen können. ${ }^{7}$ Die Historikerin Anna Green hat versucht, dieses Spannungsverhältnis konstruktiv aufzulösen, indem sie darauf hinwies: “[...] the interesting issue is not that individuals draw on contemporary cultural discourses to make sense of their lives, but which ones, and why." ${ }^{8}$ Was die Bedeutung von Erinnerungen für die Erfordernisse einer gegenwärtigen Identitätsbestimmung angeht, so lässt sich im Falle der NS-Zwangsarbeit sagen, dass der Orientierungswert der entsprechenden Erinnerungen für ein heutiges Selbstverständnis bei den meisten Interviewten dieses Untersuchungssamples vergleichsweise gering zu veranschlagen ist, mit den beiden wichtigen Ausnahmen der ukrainischen Zivildeportierten und der französischen Zwangsrekrutierten des „Service du Travail Obligatoire“ (STO), für die die Zeit der NS-Zwangsarbeit durch den jeweiligen gesellschaftlichen erinnerungskulturellen Kontext bedingt noch vergleichsweise „heiße Erinnerung“ (Charles Maier) ist.

Entschieden gegen die Behauptung einer absoluten Diskontinuität zwischen erlebter Vergangenheit und biographischer Erinnerung hat sich jüngst auch der Schweizer Historiker Christoph Dejung ausgesprochen: „Die Erfahrungen zahlreicher Oral-History-Untersuchungen legen jedoch nahe davon auszugehen, dass sich trotz aller erinnerungsbedingter Verschiebungen und mentaler Selektionsbedingungen bereits im Moment der Wahrnehmung aus den Erzählungen von Zeitzeugen zumindest eine Spur vergangener Realität herauslesen lässt.“9 Diese Spur bildet m.E. den Ausgangspunkt einer Ereigniskurve (analog zum eng-

6 Interview mit Reinhart Koselleck, in: Berliner Zeitung vom 7.5.2005, [http://www.berlinerzeitung.de/archiv/der-historiker-reinhart-koselleck-ueber-die-erinnerung-an-den-krieg--sein-endeund-seine-toten-ich-war-weder-opfer-noch-befreit,10810590,10282138.html], eingesehen 16.7.2012.

7 Christoph Dejung, Oral History und kollektives Gedächtnis, in: GG 34 (2008), S. 96-115, hier S. 98 ff. u. 115.

8 Anna Green, Individual Remembering and „Collective Memory“: Theoretical Presuppositions and Contemporary Debates, in: Oral History Journal 32 (2004) 2, S. 35-44, hier S. 42.

9 Dejung, Oral History, S. 102. 
lischen Begriff trajectory), die sich bis in die Gegenwart fortschreibt, obwohl die Qualität ihrer Repräsentation unterwegs gewissen Veränderungen unterliegt. ${ }^{10}$ Dennoch ist diese Repräsentation nicht nur von gegenwärtigen Bedingungsgefügen dominiert, sondern transportiert gleichermaßen einen in der Vergangenheit entstandenen Wahrnehmungs- bzw. Bedeutungsüberschuss mit, der als kognitive und emotionale Herausforderung in die Gegenwart hineinragt. Ebenso gibt es keinen zwingenden Grund, warum diese Ereigniskurve nicht theoretisch als Ganze zugänglich bleiben könnte, wenn auch mit stark unterschiedlichen Graden von Bewusstheit, die dann individuell als willkürliches Erinnern, spontanes Erinnern, aktives Verdrängen oder passives Vergessen erlebt werden. Es können aus aufmerksamkeitsökonomischen Gründen zwar immer nur bestimmte Auschnitte dieser Ereigniskurven aktualisiert werden, die aber auch nicht immer dieselben und auch nicht unbedingt immer die jeweils jüngsten sein müssen. Eine so verstandene Erinnerung, die aus unendlichen Schnittstellen zahlloser Ereigniskurven ihre in der Totalität unüberschaubare Komplexität erhält, ist als nicht-linearer, nicht-chronologischer Möglichkeitsraum des Erinnerns zu begreifen. Innerhalb dieses Möglichkeitsraums sind Ereignisse nicht mehr in ihrer einzigartigen Verdichtung als historisches Geschehen abrufbar; sie werden stattdessen in der Form von Ausschnitten individuell verzeitlichter, immer komplexer werdender Ereigniskurven, an die sich zunehmend Emotionen und Deutungsmuster anlagern, repräsentierbar, in denen das zugrunde liegende Ereignis als nicht mehr rückholbarer Ursprung im Hegelschen Sinne aufgehoben ist. Aus der Arbeit mit diesen Quellen erhält man zwar streng historiographisch betrachtet keine gesicherten Erkenntnisse, aber dennoch in den meisten Fällen subjektiv wahrhaftige, an den damaligen Gegebenheiten orientierte Annäherungen an historische Verhältnisse, die unser Verständnis von diesen Verhältnissen nichtsdestoweniger erweitern und differenzieren helfen.

\section{Arbeit in der NS-Zeit aus der Sicht von KZ-Häftlingen, Kriegsgefangenen und Zivildeportierten}

Der Historiker Klaus Tenfelde hat zur Frage individueller Werthaltungen gegenüber Arbeit angemerkt: "It is by the values prevailing within a given culture and

10 Mit ähnlichen Begrifflichkeiten argumentiert: Aleida Assmann, Der lange Schatten der Vergangenheit. Erinnerungskultur und Geschichtspolitik, München 2006, S. $132 \mathrm{ff}$. 
by the dominant tendencies and contradictions of the cultural mainstream that opinions about work are shaped, almost regardless of the factual nature of such work." ${ }^{11}$ Die korrespondierenden Deutungsmuster seien dabei über die Zeiten hinweg veränderlich. Das führt mitten in die Interpretationsansätze des vorliegenden Beitrags hinein. An dieser Stelle wird zunächst das Verständnis von Arbeit, das in den Erzählungen ehemaliger Zwangsarbeitender zutage tritt, näher untersucht. Die Bestimmung zur Arbeit erhielt während der NS-Herrschaft aus Sicht der Opfer wie der Täter gleichermaßen eine überragende Funktion für das Überleben der Betroffenen. ${ }^{12}$ Dies betrifft in erster Linie aus rassistischen Gründen verfolgte ehemalige Arbeitshäftlinge - in deutlicher Mehrheit Juden, dazu Sinti bzw. Roma verschiedener Nationalitäten - gilt aber in ähnlicher Form auch für die aus politischen Gründen und solchen der nationalen Zugehörigkeit Verfolgten ebenso wie für sowjetische Kriegsgefangene und schließlich auch für zahlreiche zivile Deportierte, deren Umstände der Rekrutierung und des Transports nach Deutschland ebenfalls in vielen Fällen nur geringen Zweifel daran ließen, dass ihnen kein besonders positives Schicksal von NS-Deutschland zugedacht war. Ein durchlaufender Tenor in den Interviews ist die Betonung der psychischen Einstellung, die dem Geschehen in den Lagern und an den Arbeitsstätten entgegengebracht werden musste: Als grundlegend schildern viele ehemalige Arbeitshäftlinge, dass sie die Hoffnung auf das Überleben bewahrten. ${ }^{13}$ Arbeitshäftlinge eigneten sich zudem vielfach eine Perspektive an, die als Überlebenswahrnehmung beschrieben werden könnte: Sie versuchten, die ihnen in der zunächst meist fremden Sprache zugerufenen Kommandos als einzuhaltende Spielregeln zu betrachten, sich im Arbeitsalltag möglichst unsichtbar machen, um am besten gar keine Aufmerksamkeit zu erregen oder zumindest nicht unangenehm aufzufallen, versuchten ihre Wahrnehmung zu einer gewissermaßen mentalen Selbstverteidigung ausschließlich auf ihr unmittelbares Umfeld $\mathrm{zu}$ fokussieren und erlebten schließlich an sich selbst eine stufenweise Routinisierung täglich abgerufener Handlungsabläufe. ${ }^{14}$

Ein anderer Aspekt ist die biographisch kontinuitätsstiftende Funktion der Arbeitserfahrung, die regelmäßig dort von Interviewten dargestellt wird, wo die Wahrnehmungs- und Verhaltensmuster aus Vorkriegs- und Kriegszeit einen

11 Klaus Tenfelde, Forced Labour in the Second World War: The German Case and Responsibility, in: Jürgen Kocka (Hrsg.), Work in a Modern Society. The German Historical Experience in Comparative Perspective, Oxford/New York 2010, S. 131-152, hier S. 133 f.

12 Richard Donkin, The History of Work, Basingstoke/New York 2010, S. 235.

13 Transkription des IFLDP-Interviews mit Henry G. (USA 2006), S. 49; Transkription des IFLDP-Interviews mit Anita L. (England 2006), S. 29.

14 Z. B.: Transkription des IFLDP-Interviews mit Jerzy C. (England 2006), S. 5. 
Zusammenhang bilden, der zwar durch die Verbringung nach Deutschland zunächst gewaltsam unterbrochen wurde, aber im Anschluss von den Vollzügen körperlicher (Arbeits-)Handlungen her betrachtet sich selbst in gewisser Weise ähnlich blieb. ${ }^{15}$ Diese Vorstellung wird narrativ vor allem bedeutsam, wenn Arbeit, auch in erzwungener Form, als Normalität stiftendes und Kontinuität schaffendes Element für die Konstruktion des Lebenslaufs insgesamt eine Rolle spielt. Sie übernimmt dann auch die Funktion, mit Deportation und Zwangsarbeit einhergegangene traumatische Belastungen oder fortwirkende Empfindungen der Erniedrigung und Beleidigung, von Trauer und Verlust, narrativ einzuhegen und mit einem allgemein verständlichen Vokabular alltäglicher Tätigkeitsvollzüge zu artikulieren bzw. diskursiv zu umstellen, um sie damit ihrem tatsächlich erlebten Inhalt nach ggf. umgehen zu können. ${ }^{16}$ Die Erzählung von der Arbeit erhält so eine Schutz- und Brückenfunktion, die Risse und Brüche in der Autobiographie ungesagt bleiben lässt, ohne beim Zuhörer den Eindruck einer Lücke zu hinterlassen. ${ }^{17}$

Näher an die unmittelbare Erfahrungsperspektive der Kriegszeit angelagert sind Einschätzungen, nach der selbst schwere Arbeit den Vorteil hatte, einen Fokus außerhalb von einem selbst und entfernt von der Misere des Lagerlebens zu schaffen, wie dieser englische ehemalige Arbeitskriegsgefangene schildert: "Work was hard, but it kept your mind busy." ${ }^{18}$ Dieser Effekt gilt auch über die Kriegszeit hinaus, selbst unter den deutlich gewandelten Vorzeichen der Nachkriegszeit im jeweiligen Herkunfts- oder Aufnahmeland, wenn nämlich ehemals Zwangsarbeitende nunmehr eine neu aufgenommene zivile Arbeit als Hilfestellung dafür ansahen, entweder die Kriegserfahrungen in den Hintergrund zu drängen oder durch die Arbeit ein vielfach zerstörtes Empfinden eines zivilen Alltags wieder aufzubauen. ${ }^{19}$

15 Z.B.: Umformatierte Übersetzung der Transkription des IFLDP-Interviews mit Petro K. (Ukraine 2005); umformatierte Übersetzung der Transkription des IFLDP-Interviews mit Wolodymyr M. (Ukraine 2005); umformatierte Übersetzung der Transkription des IFLDP-Interviews mit Sofia P. (Ukraine 2005); Transkription des IFLDP-Interviews mit Josef B. (Deutschland 2005), alle passim.

$16 \mathrm{Zu}$ diesem Mechanismus auch: Ulrike Jureit, Patterns of repetition: dimensions of biographical memory, in: Trauma Research Newsletter 1, Hamburger Institut für Sozialforschung, Juli 2000, S. 4 des Artikels.

17 Z. B.: Interview der Forschungsstelle für Zeitgeschichte Hamburg - Werkstatt der Erinnerung (FZH-WdE) - Projekt „In Deutschland lebende ehemalige Displaced Persons und Zwangsarbeiter“ - mit W. S. (Deutschland 1995), FZH/WdE 342, passim.

18 Imperial War Museum - Sound Archive (IWM-SA) Interview mit Alan W. (England 1996), Tonband 9. Hierzu auch: Donkin, History, S. 236.

19 Z. B.: IWM-SA Interview mit Denis A. (England 2001), Tonband 10. 
Wichtig für das erzählte Verständnis von Arbeit ist in den Interviews auch, dass zwischen der Arbeit als täglicher Beschäftigung einerseits und den Lebensbedingungen in den lagerförmigen Unterkünften samt Ernährung und hygienischer Standards sowie der gesundheitlichen Versorgung als Ensemble von Begleitumständen andererseits differenziert wird. ${ }^{20}$ Diese Differenzierung findet vor allem dort statt, wo eine eigentlich akzeptable oder sogar gute Arbeit schlechten oder gar lebensgefährdenden Rahmenbedingungen gegenüberstand. Das führte im Extremfall dazu, dass ehemalige Zwangsarbeitende nach Kriegsende wieder oder weiterhin an ihrer vorherigen Zwangsarbeitsstelle tätig waren, aber nun ihre Arbeit als „normale“ Angestellte versahen. ${ }^{21}$ Umgekehrt wirkte sich auch eine private, als menschenwürdig empfundene Unterbringung und Behandlung auf die Erfahrungsbildung, mindestens aber die spätere Verarbeitung - selbst schwerer oder körperlich schädlicher - Zwangsarbeit mildernd oder sogar positiv aus.

\section{Kategoriale Zugriffe 1: (Vernichtungs-)Haft und Lebensbedrohung}

Im Folgenden sollen verschiedene Formen von Zwang und Bedrohung, die mit der Arbeit für NS-Deutschland einhergingen, in einen kategorialen Deutungszusammenhang gestellt werden. Wiederum Tenfelde hat darauf hingewiesen, dass der Zwang als Begleiterscheinung der Arbeit in Abhängigkeit von traditionellen Loyalitätsbindungen, kulturellen Wahrnehmungs- und Deutungsmustern, persönlichen Erfahrungen sowie potentiell erwarteter Verbesserungen der persönlichen Lebensumstände verstanden werden muss. ${ }^{22}$

Dieser Aspekt ist im Falle der Arbeitshäftlinge vor allem durch die Einbeziehung des Todes als feste Größe in alltägliche Orientierungsleistungen und (Über)Lebensentscheidungen gekennzeichnet. Es war diese Art von Zwangskonstellation, in die sich hauptsächlich die Juden Europas gestellt sahen, in der sie ständig kleinräumige Verhaltensweisen entwickeln und ausprobieren mussten, um mit einer Situation umzugehen, die den Tod als manifeste Möglichkeit enthielt. Um diese Situation wiederum mental und emotional als Dauerzustand

20 Z. B.: Transkription des IFLDP-Interviews mit Wasyl B. (England 2006), S. 2.

21 Z. B.: die Transkription des IFLDP-Interviews mit Jaromir B. (England 2006), S. 35; Transkription des IFLDP-Interviews mit Anastasija S. (Deutschland 2005), S. 4.

22 Tenfelde, Labour, S. 147. 
aushalten zu können, musste sie trotz ihrer unbestreitbaren Qualität als ins Endlose verlängerter Ausnahmezustand irgendwie als Alltag imaginiert bzw. vergegenwärtigt werden. Das Narrativ des NS-Zwangsarbeitenden als „AntiHeld“ eines auf Vernichtung zulaufenden Extremalltags ${ }^{23}$ erfährt darin ebenso seine Zuspitzung wie das des schließlich aus der „tödlichen Schule des Lebens“24 zurückgekehrten, voll trotzigen Stolzes Überlebenden. Neben diesen subjektiv vollkommen verständlichen - narrativen Dramatisierungen finden sich aber vielfach eher nüchterne Protokolle des verzweifelten, mit erheblichen Kosten für körperliche Gesundheit und emotionales Gleichgewicht erkämpften Durchstehens ${ }^{25}$ sowie Berichte permanent vom Scheitern bedrohter Versuche einer Rekonstruktion des eigenen biographischen Zusammenhangs ${ }^{26}$ als Langzeitfolgen. Arbeit als existentiell erfahrenes Medium von Vernichtung ${ }^{27}$ oder Überleben $^{28}$ wird vorrangig von jüdischen Überlebenden, von sowjetischen Kriegsgefangenen sowie von Sinti und Roma artikuliert. Die deutliche Spur des Todes, die der NS-Zwangsarbeit - vor allem in ihren in den Konzentrationslagern ausgeprägten Formen - eingezeichnet ist, wird in den mündlichen Zeugnissen sehr deutlich, wie in den Worten dieses Sinto, der verschiedene Konzentrations- und Arbeitslager überstand: „Von der heutigen Generation werden wir, die einst $\mathrm{zu}$ vernichtenden Menschen, Zwangsarbeiter genannt. Ein jeder Mensch muss arbeiten, um leben zu können. Uns wurde die Zwangsarbeit auferlegt, um das tierische Krepieren zu beschleunigen. “29

Auf der anderen Seite wird der „Raum des Sagbaren“ der NS-Zwangsarbeit von der Problematisierung von Zwangs-, aber auch speziell Häftlingsarbeit als Form des fragwürdigen Ausnutzens von Begünstigungen durch die Betroffenen, das in den Bereich der Kollaboration hineingereicht habe, begrenzt. Artikuliert wird dies z. B. im expliziten Anschluss an die Schriften von Primo Levi und ist

23 Hierzu z. B.: Übersetzung der Transkription des IFLDP-Interviews mit Arie P. (Israel 2005), S. 183.

24 Transkription des IFLDP-Interviews mit Reinhard F. (Deutschland 2005), S. 32.

25 Hierzu z. B.: Transkription des IFLDP-Interviews mit Pinchas G. (Südafrika 2006), passim; British Library - National Sound Archive (BL-NSA) Interview - Projekt „Holocaust Survivors’ Centre Interviews“ - mit Manfred H. (England 1999), passim.

26 Hierzu z. B.: Transkription des Interviews des Instituts für Geschichte und Biographie (IGB) - Mauthausen Survivors' Documentation Project (MSDP) - mit Aron B. (Deutschland 2002), passim.

27 Z. B.: Übersetzung der Transkription des IFLDP-Interviews mit Uri C. (Israel 2005), S. 41.

28 Z. B.: Transkription des IGB-Interviews - Projekt „Die volkseigene Erfahrung“ - mit Aron A. (Deutschland 1987), S.14; Übersetzung der Transkription des IFLDP-Interviews mit Arie P. (Israel 2005), S. 67 u. 74.

29 Transkription des IGB (MSDP)-Interviews mit Reinhard F. (Deutschland 2002), S. 91. 
von einer stark moralisierenden Perspektive geprägt, die sich in einzelnen Erzählungen vor allem derjenigen Interviewpartner wiederfindet, die sich schon vor dem Erinnerungsboom der 2000er Jahre in Interviews geäußert haben, wie dieser deutsch-jüdische Überlebende im Jahr 1994: „(E)r [Primo Levi] bezeichnet uns als korrupt, daß wir am Leben geblieben sind, eh, haben jeden Vorteil ausgenutzt, um etwas mehr zu essen zu kriegen, und so weiter und so fort, um am Leben zu bleiben. Und so ist es wirklich gewesen. ${ }^{\text {" }}{ }^{30}$ Diese Selbst- und Fremdeinschätzung ist in all ihrer Schärfe von einem tiefreichenden Unverständnis und einem Schuldgefühl grundiert, das viele Überlebende angesichts des millionenfachen Lagertodes empfanden bzw. empfinden. Im Kontrast dazu gibt es auch jüdische Überlebende, die im Interview jegliche Versuche, der Vernichtung durch Formen von Anpassung zu entgehen, als untauglich zurückwiesen. Sie widersprachen der häufigen Aussage, die Arbeit sei ein Mittel gewesen, um sich zu retten..$^{31}$ In einer selteneren, konkurrierenden Deutung wiederum beschrieben Interviewte das Verhältnis von Arbeit und Vernichtung als ausschließlich von zeitabhängigen Nützlichkeitserwägungen der Deutschen geprägt. Bestehende Aporien des in der historischen Forschung inzwischen als problematisch wahrgenommenen Paradigmas der „Vernichtung durch Arbeit“32 ließen demnach eher ein kontingentes Verhältnis von Arbeit und Vernichtung erkennen als die ideologische oder ökonomische Rationalität eines Leitprinzips. So urteilte auch ein polnisch-jüdischer ehemaliger Arbeitshäftling, dass die Deutschen ihn so lange am Leben ließen, wie sie seine Arbeitskraft benötigten und das Kriegsende für ihn glücklicherweise diesen Wettlauf mit der Zeit beendete. ${ }^{33}$

Potentiell auch durch diese ambivalente Spannung zwischen Arbeit, Anpassung und Überleben bedingt, ist es daher oft nicht einmal so sehr die Arbeit an sich, der die Interviewten lebensrettende Wirkung zuschreiben, sondern es sind eher die konkreten Umstände ihrer Erbringung oder auch die für diese Umstände unmittelbar verantwortlichen Personen. Erinnerungen an Arbeit, Zwang und Tod haben sich in diesen Kontexten gegenseitig verstärkt und reichen seitdem als Bedeutungsüberschuss in die späteren Deutungsbemühungen

30 Transkription des IGB-Interviews - Projekt „Wiedergutmachung“ - mit Hans F. (Deutschland 1994), S. 93. Hierzu z.B. auch: Übersetzung der Transkription des IFLDP-Interviews mit Uri C. (Israel 2005), S. 82.

31 Z. B.: BL-NSA Interview - Projekt „Holocaust Survivors’ Centre Interviews“ - mit Manfred H. (England 1999), Kassette 2, Seite A; Übersetzung der Transkription des IFLDP-Interviews mit Uri C. (Israel 2005), S. $54 f$.

32 Donkin, History, S. 198.

33 Z. B.: BL-NSA Interview - Projekt „Millennium Memory Bank“ - mit Sam N. (England 1999), MP 3, passim. 
hinein. Die verschiedenen, von den Überlebenden eingeschlagenen Wanderungswege nach dem Krieg scheinen sich hingegen auf diesen speziellen Ausschnitt der Erinnerungsbildung vergleichsweise wenig spezifisch ausgewirkt zu haben, da sie länderübergreifend bei zurückgekehrten und ausgewanderten ehemaligen Zwangsarbeitenden in ähnlicher Form anzutreffen sind.

\section{Kategoriale Zugriffe 2: (Kriegs-)Gefangenschaft und Statusbedrohung}

Ein eher untergeordneter Stellenwert kommt der Arbeit in denjenigen Interviews - hauptsächlich mit ehemaligen Arbeitskriegsgefangenen, allerdings auch in signifikanter Weise mit Arbeitshäftlingen - zu, in denen sie zwar erwähnt wird und auch durchaus im Gang der Erzählung einigen Raum einnimmt, sich allerdings schon zum Zeitpunkt der Erfahrung andere politische, soziale oder kulturelle (Selbst-)Wahrnehmungen und Deutungen als relevanter erwiesen haben. ${ }^{34}$ So findet sich besonders in den Erzählungen britischer Arbeitskriegsgefangener eine dezidierte biographische Herabstufung der geleisteten Zwangsarbeit, die vielfach der Erfahrung der Gefangenschaft im Lager und dem Status als internierter Kombattant untergeordnet wird. ${ }^{35}$ Vordringlich wird dabei das Empfinden herausgestellt, trotz widriger und teils auch brutaler Bedingungen noch ein leichteres Schicksal als z. B. sowjetische Kriegsgefangene, Polen und Juden, die dann je nach den konkreten Umständen auch in abgrenzender Absicht explizit als Zwangsarbeiter bezeichnet werden, gehabt zu haben. ${ }^{36}$ Die Arbeit erfüllte nichtsdestoweniger auch für diese Interviewten eine Reihe biographischer bzw. narrativer Funktionen: So wurde die Arbeit zu einem räumlichen Orientierungspunkt für solche überlebenden ehemaligen Zwangsarbeitenden, die häufig ihren Internierungs- und Arbeitsort wechseln mussten. Da sie von den jeweiligen Örtlichkeiten kaum einen bleibenden Eindruck gewinnen konnten, waren es eher die wechselnden Tätigkeiten, die von ihnen verlangt wurden, die den Erinnerun-

34 Z.B.: Transkription des IGB-Interviews - Projekt „Wiedergutmachung“- mit Hans F. (Deutschland 1994), passim.

35 Z.B.: ebd.

36 Z. B.: IWM-SA Interview mit Joseph B. (England 1995), Tonband 1, Tonband 2 u. passim; IWM-SA Interview mit Bernard P. (England 1980), Tonband 4; IWM-SA Interview mit Ernest T. (England 1996), Tonband 7. 
gen Kohärenz verleihen. ${ }^{37}$ Die Arbeit wurde auch wiederholt in einer schlichten Zweck-Mittel-Relation gesehen, wenn sie nämlich eine bessere Ernährung ermöglichte, unter annehmbareren hygienischen Verhältnissen stattfand oder einen im Vergleich zum Lager oder zum vorigen Arbeitsplatz unter das Kommando erträglicherer Aufseher oder Vorarbeiter brachte. ${ }^{38}$

Der Zugriff dieser Kategorie ist zwar zunächst definitionsgemäß auf die Gruppe der Arbeitskriegsgefangenen zugeschnitten, von denen im Interviewsample dieses Beitrags aufgrund der Entstehungsgeschichte des IFLDP hauptsächlich ehemalige britische Kriegsgefangene vertreten sind. ${ }^{39}$ In ihrer Situation trafen zwei Dynamiken aufeinander, die ihnen einerseits eine Scharnierfunktion zwischen den Zwangsarbeitserfahrungen ehemaliger Arbeitshäftlinge und der als „Ostarbeiter“ bezeichneten Arbeitsdeportierten zuschreiben, die es andererseits aber auch fast unmöglich machen, sie überhaupt als Gruppe zusammenzufassen. Sie hatten kraft ihres Status als Kriegsgefangene den Vorteil, dass das meiste, was ihnen widerfuhr, theoretisch von einem international anerkannten Regelwerk und von einschlägigen historischen Vorerfahrungen gerahmt war. Insofern war die Bedrohung dieses Status für sie zunächst die einzige unmittelbar relevante Gefährdung. Gleichzeitig wurde dieser Rahmen aber auch in einer Vielzahl von Fällen so eklatant missachtet oder gesprengt, dass es notwendig erscheint, den zunächst vergleichweise harmlos klingenden Begriff „Statusverlust" bis hin zur unmittelbaren Lebensbedrohung neu zu dimensionieren und in seiner Bedeutung auch auf andere Gruppen als die britischen Kriegsgefangenen auszudehnen. Die Dramatik dieses Statusverlustes wurde besonders im Fall der ehemaligen sowjetischen Kriegsgefangenen und später auch der italienischen Militärinternierten deutlich, gegenüber denen sich Deutschland nicht an die internationalen Regelungen gebunden fühlte und deren Behandlung zudem von rassistischen und politischen Ideologemen unterfüttert war.

37 Z. B.: Transkription des IGB-Interviews - Projekt „Die volkseigene Erfahrung“ - mit Aron A. (Deutschland 1987), passim.

38 Z. B.: IWM-SA Interview mit Joseph B. (England 1995), Tonband 1; IWM-SA Interview mit Leslie E. (England 1998), Tonband 2.

39 Aufgrund des Ausschlusses von Kriegsgefangenen aus dem Geltungsbereich des Zwangsarbeiterentschädigungsgesetzes waren sie im IFLDP nur in relativ geringer Zahl vertreten. Um die Erfahrungen dieser Gruppe stärker repräsentieren zu können, wurden vom Autor zusätzlich 11 Interviews des Imperial War Museum - Sound Archive (IWM-SA) bzw. des National Sound Archive der British Library (BL-NSA, beide in London) mit dem entsprechenden Personenkreis herangezogen. Außerdem wurden die sogenannten „Freitagsbriefe“ des Berliner Vereins „KONTAKTE- KOHTAKTbI e.V.“, in denen ehemalige sowjetische Kriegsgefangene ihre Erinnerungen beschreiben, regelmäßig ausgewertet: [http://www.kontakte-kontakty.de/deutsch/nsopfer/freitagsbriefe/], eingesehen 26.1.2014. 
Aus den Schilderungen der spezifischen Bedrohungslage der britischen ehemaligen Arbeitskriegsgefangenen werden zudem eine Reihe, teilweise langzeitwirksame Beeinträchtigungen erkennbar. Das innerlich als identitätsgefährdend erlebte Aufgehen im Massenschicksal, exemplifiziert am täglich wiederholten, als unerträglich empfundenen Lagerdasein, tritt aus ihrem Blickwinkel heraus ebenso prononciert hervor wie das als ständig fortlaufender Kampf geschilderte Bemühen um die Bewahrung des angestammten Soldatenstatus, in dem auf Abweichungen rechtlicher Garantien mit spontanem, als selbstverständlich erachtetem Protest oder ungläubiger Hinnahme reagiert wurde. ${ }^{40}$ Beide Aspekte zusammengenommen bündelten sich für sie in der ständigen Bedrohung mit der Verfügbarmachung für fremdbestimmte, aufgenötigte Zwecke (zumeist willkürlich zugeteilte Arbeitsleistungen), jedoch erzeugten diese auch schon seinerzeit bzw. im Prozess der Verarbeitung die subjektive Notwendigkeit, sich schamvoll von anderen Statusgruppen, die vergleichsweise schlimmeren Umständen ausgesetzt waren, abzugrenzen. ${ }^{41}$

\section{Kategoriale Zugriffe 3: Körperliche Dislozierung und Identitätsbedrohung}

Unter den ehemaligen Arbeitsdeportierten beschreiben einige die Zwangsarbeit im Nachhinein durchaus nüchtern als eine vergleichsweise erträgliche Konstellation, um den Krieg zu überstehen. Diese Einschätzung beruht einerseits auf dem Vergleich mit der Situation der kämpfenden Frontsoldaten, andererseits aber vor allem in Osteuropa auch auf der Erfahrung bzw. später erworbenen Kenntnis des harten Besatzungsalltags. Gleichzeitig machte und macht sie das aber auch in ihren jeweiligen Herkunftsgesellschaften angreifbar für Vorwürfe wegen „Vorteilsnahme durch Kollaboration“. ${ }^{42}$ Darüber hinaus waren es auch für die Arbeitsdeportierten eher bestimmte Tätigkeitsformen oder die individuelle Art der Durchführung der Arbeit, die ihnen Hoffnung aufs Überleben gaben. Das wurde in Interviews besonders dann zum Ausdruck gebracht, wenn in der Arbeit die beruflichen Vorkenntnisse oder Interessen der Zwangsarbeitenden zur Geltung kommen konnten, wie diese tschechische ehemalige Arbeitsdeportierte zu erkennen gibt: „Ich war vielleicht technisch 'n bisschen begabt. Das

40 Z. B.: IWM-SA Interview mit Bernard P. (England 1980), Tonband 1 u. 3.

41 Z. B.: IWM-SA Interview mit Alan W. (England 1989), Tonband 3 u. 9.

42 Z.B.: Übersetzung der Transkription des IFLDP-Interviews mit Boleslav W. (Tschechien 2005), S. 34. 
dauerte nich' lange, da durfte ich denn die Maschinen einrichten, die [...] Bohrer einbauen und die Bohrer schleifen, wie gesagt, das hab' ich dann auch alles getan, ja. “43 Den daraus ableitbaren Stolz auf die arbeitstechnischen Fertigkeiten, der sich als Schlüsselressource für das Selbstwertgefühl, den Überlebenswillen und die kognitive Entschlüsselung der Zwangsarbeit als zeitlich bedingte Überlebenschance erweisen konnte ${ }^{44}$, artikulierten am ehesten tschechische Interviewpartner, wenn auch selten so deutlich wie der folgende: „[...] da habe ich gesehen, dass das alles supermoderne Maschinen sind und meist hat niemand anders daran, an diesen Maschinen, gearbeitet als Tschechen. Also waren wir nicht nur irgendein Volk, das, wir waren ein Volk, das etwas konnte.“45

Die tschechischen Zwangsarbeitenden repräsentieren einerseits eine gewisse Bandbreite von Zwangsarbeitserfahrungen, die andererseits vergleichsweise homogene narrative Ausdrucksformen gefunden haben. Dadurch stellen sie eine Verbindung zwischen den Erfahrungsräumen der ost- und der westeuropäischen Arbeitsdeportierten her, deren Statusunterschiede und entsprechenden Unterschiede in den Arbeitserfahrungen doch beträchtlich waren. Zudem machte ein individuell qualifizierter, maschinengestützter Einsatz die Arbeit der Zwangsarbeitenden generell höherwertiger und sie wurden gleichzeitig weniger leicht ersetzbar. Es stärkte also ihre Position im Produktionsablauf und führte dazu, dass sich Handlungsspielräume ergaben, teilweise sogar für angebliche oder tatsächliche Sabotagehandlungen. ${ }^{46}$ Bei Kolonnenarbeiten mussten Zwangsarbeitende eher darauf hoffen, im Rahmen einer generell schweren Arbeit durch pures Glück eine leichtere Teilaufgabe zugewiesen zu bekommen. Es gab auch, sofern der Überwachungsdruck nicht durchgängig gegeben war, zumindest temporäre Gelegenheiten, das Arbeitstempo oder die $\mathrm{zu}$ bewegenden Lasten $\mathrm{zu}$ verringern, bevor die Betreffenden unter Beobachtung wieder den oft willkürlich überhöhten Ansprüchen der Aufseher genügen mussten. ${ }^{47}$

Als Gesamtgruppe betrachtet betonen die interviewten ehemaligen Arbeitsdeportierten stärker solche Gefährdungslagen, in denen sich die Deportation

43 Transkription des IGB-Interviews - Projekt „Wiedergutmachung“ - mit Ilse S. (Deutschland 1994), S. 4. Vgl. auch: Übersetzung der Transkription des IFLDP-Interviews mit Ladislav M. (Tschechien 2006), S. 6.

44 Jacob Goldstein etal., Individuelles und kollektives Verhalten in Nazi-Konzentrationslagern. Soziologische und psychologische Studien zu Berichten ungarisch-jüdischer Überlebender, Frankfurt a.M./New York 1991, S. 32ff.

45 Übersetzung der Transkription des IFLDP-Interviews mitLadislavM. (Tschechien 2006), S. 49.

46 Z. B.: Übersetzung der Transkription des IFLDP-Interviews mit MarieJ.(Tschechien 2005), S.9.

47 Z. B.: BL-NSA Interview - Projekt „Holocaust Survivors’ Centre Interviews“ - mit Manfred H. (England 1999), Kassette 1, Seite B. 
und die Zwangsarbeit aktuell oder langfristig destabilisierend auf individuelle Selbstverständnisse und sozialräumliche Zugehörigkeiten ausgewirkt haben, da Todesgefahr für sie oft weniger konkret erfahrbar war und kein spezifischer Statusverlust drohte. Diese im Verhältnis zur ersten Gruppe also zunächst deutlich geringer unmittelbar existenzgefährdend wirkenden Bedrohungen konnten jedoch vor allem im Fall von Jugendlichen subjektiv dennoch sehr dramatische Formen annehmen. Insofern ist hier einerseits eher von einer generationell, nur bedingt national $\mathrm{zu}$ differenzierenden Gefährdungslage $\mathrm{zu}$ sprechen, wobei jedoch andererseits $\mathrm{zu}$ berücksichtigen ist, dass die jugendlichen Zwangsarbeitenden überwiegend aus der ehemaligen Sowjetunion kamen.

Diese spezifische Bedrohungslage weist auch allgemein auf prekäre Übergangszustände der Kriegs- und Nachkriegszeit hin, die in ihrer Unübersichtlichkeit und Widersprüchlichkeit besonders von den britischen ehemaligen Arbeitskriegsgefangenen, den französischen ehemaligen STO-Rekrutierten sowie den in Deutschland gebliebenen ehemaligen Zwangsarbeitenden artikuliert wurden. Besonders bei der letzten Gruppe zeigen sich auch die spezifischen Auswirkungen der Migrationswege ehemaliger Zwangsarbeitender während des Krieges und danach. Denn die durch Deportation und Zwangsarbeit ausgelösten Wanderungsprozesse verliefen nicht notwendigerweise immer als langfristig geschlossene Abfolge von Trennung und Neuankunft und damit verbundener Transformation des Selbstempfindens. Die Offenheit bzw. Unabschließbarkeit dieser Prozesse haben sich in den genannten Gruppen am stärksten ausgewirkt. So erscheint der Aufenthalt in der (deutschen) Aufnahmegesellschaft teilweise eher als Ort des „Dazwischen“ bzw. das Dasein geriet schon während, spätestens aber nach Ende der Zwangsarbeit zum Leben zwischen den Welten. ${ }^{48}$ Die Kriegs- und Zwangsarbeitserfahrung konnte auch einen Einstellungswandel der Betroffenen bewirken, der das Leben danach als ständige Abfolge von Anpassungsprozessen erscheinen ließ. ${ }^{49}$ Und auch eine erfolgreiche biographische Selbstverstetigung nach dem Krieg konnte vielfach nur mit Abspaltungsleistungen gegenüber der Vergangenheit erworben werden, so dass diese entweder nur als entfernt wahrnehmbare Erfahrung räumlicher Desintegration oder als vollständiger Fremdkörper im Lebenslauf verbucht wurde. ${ }^{50}$

48 Z. B.: Transkription des FZH-WdE-Interviews - Projekt „In Deutschland lebende ehemalige Displaced Persons und Zwangsarbeiter“ - mit S. Z. (Deutschland 1994), S. 7.

49 Z. B.: Transkription des IGB-Interviews - Projekt „Die volkseigene Erfahrung“ - mit Roman K. (Deutschland 1987), passim.

50 Z. B.: Transkription des IFLDP-Interviews mit Gabriel F. (Frankreich 2006), S. 25; übersetzte Transkription des IFLDP-Interviews mit Victor L. (Frankreich 2006), passim. 


\section{Individuelle Wege der biographischen Integration}

Die im vorigen Satz angedeutete, eher defensive Möglichkeit der Verarbeitung besteht in einer mentalen „Auftrennung“ der zeitlichen Dimensionen der seinerzeitigen und der gegenwärtigen Erfahrung, durch die die absolute Diskontinuität zwischen dem damaligen und dem heutigen Geschehen betont wird. Die Zeit der Zwangsarbeit wird so zum gänzlich ,Anderen“ der Gegenwart, das für diese nicht mehr bedrohlich werden kann, aber auch emotional und kognitiv nur noch aus großer Entfernung beschrieben wird und subjektiv stark an Bedeutung verloren hat, wie in den Worten dieses nach England ausgewanderten tschechischen ehemaligen Arbeitsdeportierten: “And, it's completely different, you know. I got friends, and when you come there, they tell you all the time the same story, every time the same things, what's happened during the war, he was in India and that things. I don't want to know. I want to know what'll come next week, not what was last year. And that's always the same things, I always think forward." ${ }^{51}$ Vor allem bei jüdischen Überlebenden hat sich diese Auftrennung der zeitlichen Dimensionen teilweise zu einer tief sitzenden kognitiven Unvereinbarkeit zwischen dem Leben vor 1933, der Zeit der Verfolgung, der Zeit der Vernichtung und dem Leben danach verdichtet: "So one was very, 'Das kann, ist nicht möglich, was hier passiert.' But bit by bit one realized, 'Alles ist möglich.' You know, when the Nazis took over, everything collapsed really what was normality." ${ }^{2}$

Die Ebene des Vergleichs ist insgesamt unter den Interviewten ein wesentlicher Faktor der Verarbeitung der Erfahrungen und der Deutung der Erinnerungen. So werden eigene Zwangsarbeitserfahrungen im Lichte der inzwischen vorhandenen historischen Kenntnisse, die sich auch zahlreiche ehemalige Zwangsarbeitende offensiv angeeignet bzw. zu deren Zustandekommen manche selbst aktiv beigetragen haben, nüchtern ins Verhältnis zu anderen Formen von Verfolgung und Zwangsarbeit gesetzt und dabei oft (gewollt) positiv relativiert, wie dieser deutsche ehemalige Arbeitshäftling im Bericht über seine KZ-Haft während des Jahres 1933 andeutet: „Sie haben auch noch, wie gesagt, sie hatten noch nicht erfunden (lacht verhalten) die Vernichtung durch Arbeit. Das hatten sie noch nicht erfunden. "53 Anders als die allermeisten Arbeitshäftlinge begreifen manche, vor allem osteuropäische ehemalige Arbeitsdeportierte inzwischen

51 Transkription des IFLDP-Interviews mit Jaromir B. (England 2006), S. 19.

52 Transkription des IFLDP-Interviews mit Anita L. (England 2006), S. 21f.

53 Transkription des IFLDP-Interviews mit Philipp W. (Deutschland 2006), S. 14. 
die NS-Zwangsarbeit mehr als Teil eines biographischen Kontinuums, innerhalb dessen wechselnde, mit Elementen von Zwang einhergehende Arbeitsbedingungen im Laufe des Lebens ohne spezifische ethische oder politische Kontextualisierung fortlaufend als Herausforderungen gemeistert werden mussten bzw. müssen. Darin zeigt sich ein Muster der Alltagsbewältigung oder mindestens der rückschauenden Analyse, das besonders unter ethnischen und anderen Minderheiten anzutreffen ist. Für diese war und ist es teilweise generationenübergreifende Realität, mit verschiedenen Formen von Diskriminierung und Verfolgung umzugehen, die in einen fortlaufenden Erfahrungszusammenhang integriert werden. Indem einzelne Verfolgungserlebnisse zueinander ins Verhältnis gesetzt, gegeneinander abgewogen und zu einem biographisch integrierbaren Ganzen gemacht werden, ragen einzelne Perioden für sich genommen nicht notwendigerweise heraus, sondern bekommen erst in der lebensgeschichtlichen Gesamtschau ihre Bedeutung zugewiesen. Die Erfahrung von brutaler Zwangsarbeit unter KZ-Bedingungen ist in diesem Wahrnehmungskontinuum jedoch in der Regel ein fundamentaler, herausgehobener Bezugspunkt für die Erinnerungsbildung und die biographische Integration von Arbeits- und Verfolgungserfahrungen.

\section{Fazit: NS-Zwangsarbeit als individualisierte und kollektivierte Zuschreibungsressource}

Zwangsarbeit in der NS-Zeit zeigt sich in den Interviews als heterogene, zeitlichem Wandel unterworfene Sammelbezeichnung, die vom mit knapper Not überstandenen Mordversuch durch ruinöse körperliche Überforderung bis zur vergleichsweise glimpflichen, wenn auch auszehrenden Nischenexistenz unter Kriegsbedingungen reichte und die Betroffenen gleichzeitig verschiedenen Bedrohungsszenarien von der unmittelbaren Gefährdung von Leib und Leben bis zur Destabilisierung der psychischen und räumlichen Integrität unterwarf. Dies gilt ausdrücklich nicht nur für verschiedene Statusgruppen, sondern auch innerhalb vieler individueller Lebensläufe über die Dauer des Krieges hinweg. Entsprechend wurden in den Erinnerungen ebenso weit gespannte Strategien des Umgangs mit erzwungener Arbeit in der NS-Zeit erkennbar, in denen einerseits die Konzentration auf das Überleben als essentielles mentales Element erscheint, um Identität und Durchhaltewillen zu wahren oder zu rekonstruieren. Andererseits konnte sich aber auch die hohe Funktionalität der Anerkennung am Arbeitsplatz für Überleben und Selbstempfinden als gleichermaßen bedeutsam erweisen. Solche Beobachtungen haben zudem über den engen 
biographischen Kontext hinaus auch Einfluss auf wegweisende arbeitspsychologische Forschungen in der Nachkriegszeit genommen. ${ }^{54}$

Es dominiert in den Interviews generell ein Verständnis von Arbeit, nach der sie mit körperlicher Anstrengung zusammengedacht wird und Tätigkeiten wie z. B. Nähen, selbst wenn sie zum Lebensunterhalt beitrugen, kaum als „echte" Arbeit gewürdigt werden. ${ }^{55}$ Abgesehen von der Funktion der Arbeit im Kontext der NS-Vernichtungspolitik zeigen sich nicht nur insofern in den Interviews keine Anzeichen einer prinzipiellen Problematisierung von - auch schwerer körperlicher - industrieller, landwirtschaftlicher oder anderer Arbeit an sich in ihren Auswirkungen auf die Menschen, die sie verrichten. ${ }^{56}$ Diese grundsätzlich affirmative Perspektive auf (schwere, körperliche) Arbeit bildet sich im diskursiven Extremfall bei ehemaligen Zwangsarbeitenden, die ihre Tätigkeit ohne akute Todesdrohung ausübten, in Form eines narrativierten, selbst empfundenen oder vom (realen, imaginierten) Zuhörer erwarteten Respekts für die erbrachte Leistung $a b .^{57}$ An diesen Befund anschließend soll dieses Fazit noch einen weiter gespannten gesellschaftlichen Bezugsrahmen aufnehmen, der für das Verständnis der vorliegenden Forschung auch eine wichtige Rolle spielt.

Seit den 1980er Jahren beginnt sich in den westlichen Industriegesellschaften die Sicht auf Arbeit zu wandeln. Mit der Veränderung der industriellen Arbeits- zu post-industriellen Konsumgesellschaften scheint sich gleichzeitig ein Bewusstsein auszubilden, mit dem es möglich wird, bestimmte Formen von Arbeit nicht nur abstrakt-normativ, sondern auch in den Werthaltungen der Mehrheitsgesellschaften grundsätzlich zu delegitimieren. Mit dem dabei entstandenen Diskursraum interagierte auch die Debatte um die Entschädigungen für NS-Zwangsarbeit. Der Begriff der NS-Zwangsarbeit in seiner gegenwärtigen Form hat eine empirisch legitimierte, aber in seiner politisch-moralischen Verdichtung gleichzeitig auch synthetische Diskursposition geschaffen, von der aus es möglich wurde, die industrielle Arbeitsorganisation der Kriegswirtschaft NS-Deutschlands fundamental zu diskreditieren. Oder wie es Tenfelde ausgedrückt hat: "It [die Entschädigungsdebatte, CT] has fostered a sense of victimization that aims at reconstructing the victims' experiences to enforce legal claims." ${ }^{58}$ Zwischen den dies in der Vergangenheit nachhaltig bestreitenden,

54 Donkin, Work, S. $235 f$.

55 Z. B.: umformatierte Übersetzung der Transkription des IFLDP-Interviews mit Galina G. (Ukraine 2005), S. 10; Protokoll zum IFLDP-Interview mit Jutta P. (Deutschland 2006).

56 Z. B.: Übersetzung der Transkription des IFLDP-Interviews mit Gabriel F. (Frankreich 2006), S. 31 .

57 Z. B.: Transkription des IFLDP-Interviews mit Wilhelm N. (Deutschland 2006), S. 21.

58 Tenfelde, Labour, S. 148. 
konkurrierenden exkulpierenden Erinnerungen der deutschen Bevölkerung und denjenigen der NS-Zwangsarbeitenden gibt es zwar eine gewisse Schnittmenge. Umso deutlicher ist aber umgekehrt auch auf die erheblich größere Menge wesentlicher Differenzen hinzuweisen. Es mögen nicht immer unbedingt die unmittelbaren Vollzüge der Arbeit gewesen sein, die Leid zufügten, sondern oft mehr noch die gewaltsamen Umstände, unter denen sie stattfanden. Auf Seiten der deutschen Bevölkerung scheint die Sicht auf Formen erzwungener Arbeit in der NS-Zeit im Nachhinein weniger von Verdrängung (wie lange Zeit im Fall der Shoah) geprägt gewesen zu sein, sondern eher von einer manifesten Opferkonkurrenz. Viele Deutsche wollten selbst gern einen Opferstatus für sich reklamieren; in diesem Bemühen waren Millionen internierter, gefangen genommener oder verschleppter und zur Arbeit genötigter Ausländer unerwünschte Mitbewerber. Das führte in der Konsequenz schließlich dazu, dass der deutschen Bevölkerung jahrzehntelang in vielen Fällen die Bereitschaft oder die Kapazität fehlte, das von ihnen hingenommene oder befürwortete oder sogar willkürlich herbeigeführte täglich aktualisierte Drama von Dislozierung, Identitätsbedrohung, Statusverlust oder des drohenden Todes auf Seiten der Zwangsarbeitenden in angemessener Weise erinnern und anerkennen zu können. Dieser aus subjektiver oder objektiver Opferkonkurrenz resultierende Mangel findet sich in abgewandelter, aber vergleichbarer Weise in allen untersuchten Gesellschaften wieder, was sich besonders nachhaltig für ehemalige Zwangsarbeitende aus der ehemaligen Sowjetunion und die STO-Rekrutierten aus Frankreich ausgewirkt hat. ${ }^{59}$ Eigene Besatzungserlebnisse, lebensgefährliche Fronteinsätze oder Widerstandshandlungen, verdrängte Kollaborationserfahrungen oder schlichter Neid auf die in Feindeshand Davongekommenen - all dies hat die Spielräume für soziale und erinnerungskulturelle Anerkennung ehemaliger NS-Zwangsarbeitender enorm eingeengt.

Die Verwendung lebensgeschichtlicher Interviews mit ehemaligen Zwangsarbeitenden als historische Quellen lässt es insgesamt sinnvoll erscheinen, unter den NS-Zwangsarbeitenden begrifflich zu differenzieren und streng definitorisch nur diejenigen als Zwangsarbeiter/in anzusprechen, die als Zivilpersonen - ohne vorher Partisanen- oder sonstige gegen die Besatzer gerichtete Tätigkeiten geleistet $\mathrm{zu}$ haben oder dessen verdächtigt worden $\mathrm{zu}$ sein - aus keinem anderen Grund und zu keinem anderen Zweck als zur Erpressung ihrer Arbeitsleistung nach Deutschland verbracht wurden. In allen anderen Konstel-

59 Hierzu auch: Alexander von Plato, „Es war moderne Sklaverei.“ Erste Ergebnisse des lebensgeschichtlichen Dokumentationsprojekts zur Sklaven- und Zwangsarbeit, in: BIOS 20 (2007) 2, S. 251-290, hier S. 285f. 
lationen der Verschleppung und anschließenden Nötigung zur Arbeit neigen NS-Opfer dazu, in ihren Erinnerungen dominantere Identifikationsmuster als biographische Marker und Träger eines personalen Selbstverständnisses über die als quälend, erniedrigend, lästig, oder in wenigen Fällen sogar in einem existentiellen Sinne als zufriedenstellend erlebte „Begleiterscheinung“ Arbeit zu schieben. Diese Überlebenden bezeichnen sich selbst vielfach eher als KZ-Häftlinge, Widerstandskämpfer, aus Gründen nationaler, religiöser oder sozialer Zugehörigkeit Verfolgte oder als Kriegsgefangene, die neben anderen existentiell bedeutsamen Kriegserfahrungen eben auch erzwungene Arbeitsleistungen erbringen mussten. Arbeit gehörte im 20. Jahrhundert offenbar im narrativen Kontext der biographischen Selbstpräsentation vieler Interviewter wie auch im Verständnis der jeweiligen Gesellschaften so selbstverständlich zur menschlichen Existenz, dass sie unter den meisten Umständen als ein Element der Normalität bzw. der Kontinuitätsstiftung erlebt wurde und erinnert wird. Dadurch erscheint Arbeit tendenziell ungeeignet als Element der Skandalisierung bzw. zur Ausgestaltung von Verfolgungserfahrungen, innerhalb derer sie jenseits des Bereichs der unmittelbaren physischen Existenzgefährdung - eher einem Prozess der narrativen Normalisierung unterliegt. Durch die Verwendung lebensgeschichtlicher Interviews wird so eine weitere Perspektive des historischen Verständnisses erzwungener Arbeitsleistungen in Deutschland während des Zweiten Weltkrieges eröffnet, die neben die klassisch empirische Analyse des Zwangsarbeitsstatus, die nach dem Vorhandensein der Potentiale von exit, voice und probability of survival sucht ${ }^{60}$, eine solche stellt, die die Selbstzuschreibungen dieses Status durch die Betroffenen und die erinnerungskulturellen Fremdzuschreibungen - und damit die individualisierten und kollektivierten Erfahrungskontexte und Deutungsmuster - als maßgebliche Dimensionen für eine Gesamtbewertung des Komplexes der NS-Zwangsarbeit mitberücksichtigt.

60 Mark Spoerer, Zwangsarbeit unter dem Hakenkreuz. Ausländische Zivilarbeiter, Kriegsgefangene und Häftlinge im Deutschen Reich und im besetzten Europa 1939-1945, Stuttgart 2001, S. $15 f f$. 